\title{
Metastatic paranasal sinuses adenocarcinoma from breast carcinoma, a rare event: a case report
}

\author{
Tawfiq Khurayzi $^{1 *}$, Abdullah Alshahrani ${ }^{2}$
}

\begin{abstract}
Department of Otolaryngology, ${ }^{1}$ King Fahad Central Hospital, Jizan, ${ }^{2}$ Armed Forces Hospital, South Region, Khamis Mushayt, KSA
\end{abstract}

Received: 28 May 2017

Revised: 25 July 2017

Accepted: 27 July 2017

\section{*Correspondence:}

Dr. Tawfiq Khurayzi,

E-mail: mezo106@gmail.com

Copyright: ( $)$ the author(s), publisher and licensee Medip Academy. This is an open-access article distributed under the terms of the Creative Commons Attribution Non-Commercial License, which permits unrestricted non-commercial use, distribution, and reproduction in any medium, provided the original work is properly cited.

\begin{abstract}
Metastases to the nose and paranasal sinuses usually respond poorly to treatment and have a poor prognosis. We present a 31 year old lady with rare metastatic paranasal sinuses lesion which is adenocarcinoma that metastasis from breast carcinoma. All of clinical presentation, radiological findings, histopathological and immunohistochemically markers examination reveal metastatic adenocarcinoma. This study presents rare metastasis in head and neck cancer especially from breast to paranasal sinuses.
\end{abstract}

Keywords: Metastatic tumors to paranasal sinuses, Adenocarcinoma, Metastases in breast carcinoma

\section{INTRODUCTION}

Nasal cavity and paranasal sinus cancers are usually primary tumor and accounts forabout $0.3 \%$ of all cancers. ${ }^{1}$ Distant metastatic tumors to paranasal sinuses are well documented but uncommon and exceeding lyraretype. The clinical presentation is similar to that of primary tumors and common symptoms include recurrent epistaxis, nasal obstruction and facial pain. ${ }^{2}$ Most frequently metastasis to paranasal sinuses are those that come from primary malignant as in renal cell carcinoma followed by bronchus, urogenital ridge, breast and gastrointestinal tract, respectively., ${ }^{3,4}$ Few cases of metastatic carcinoma to the paranasal sinuses as well as reported in the literatures review of the 2004-2010 pathology records of patients with sinonasal malignancy revealed three cases with distant metastases to this region from primary sites in kidney, prostate and breast. Breast cancer metastases occur in $>50 \%$ of patients. However, from breasts cancer commonly developed metastases in the body but rarely to head and neck and extremely rare to paranasal sinuses.

Sinonasal metastasis should be make in differential diagnosis with primary tumors such as adenocarcinomas, melanomas, angiofibromas, hemangiomas, metastatic tumors and systemic diseases such as Wegener's and midline granulomas. ${ }^{6}$ Finally, any patient with unfrequently sinonasal symptoms and signs with suspected malignancy he should underwent full assessment including radiological examination with CT scan and MRI and angiography if necessary and then histopathological and immune-histochemically markers examination it should be considered and it useful to confirming the origin of the neoplasm. Pathological way of metastasis to the paranasal sinuses until now it is unclear and there many controversial theories in breast carcinoma spreads both by way of blood vessels and lymphatic channels. ${ }^{7}$ We present here a case report that known case of breast cancer that metastatic to the sphenoid, ethmoidal and frontal sinuses with left orbital mass lesion. 


\section{CASE REPORT}

In 2010, 31 year-old Saudi female diagnosed with advanced stage ductal carcinoma in left breast. Left radical mastectomy with axillary regional lymph node dissection and adjuvant chemotherapy was performed at high specialty Hospital in Riyadh Saudi Arabia with regular follow up there. In 2015, she presented in Khamis Mushait Hospital, south region, at Emergency Room with left eye proptosis, left loss of vision within four days, left facial pain, bilateral nasal discharge, left nasal obstruction, and on/off left nasal bleeding for more than 4 months. On clinical examination: left eye proptosis, conjunctivitis, edema surrounding left eye, total loss of vision and tenderness. Anterior rhinoscopy and endoscopy done showed left nasal crusting secretion and congested nasal mucosa with softy red nasal mass in posterior part of left nasal cavity extended partially to nasopharynx. Rest of ENT examination is unremarkable. We referred patient to ophthalmology, oncology and general surgery clinics to assess her. We requested neck and paranasal sinus computer tomography (CT) scan and Magnetic Resonance imaging (MRI). CT showed: left nasal cavity, nasopharyngeal, left ethmoidal sinus and sphenoid sinus mass lesion. This mass it enhanced, infiltrate to be left extra coanal orbital mass lesion with multiple bone destruction included the nasal bone and posterior part of nasal septum, left frontal sinus and left lamina paparycea with medial recti muscles and surrounded the left optic nerve. The skull base was not involved and Brain tissue showed normal signal intensity (Figures 1-4).

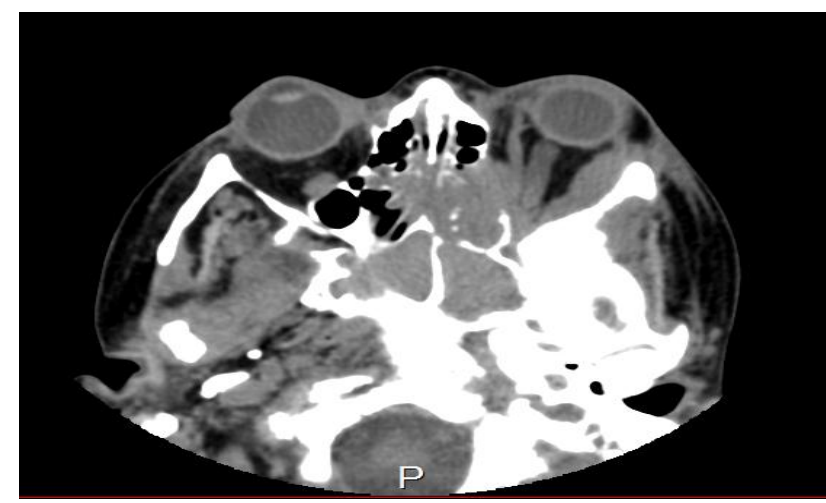

Figure 1: Axial plain brain CT shows soft tissue like lesion is originating from posterior aspect of nasal cavity extending up to the ethmoid sinuses.

After discussion with the patient, we decided to do endoscopic maxillary antrostomy, ethmoidectomy and sphenoideotomy with several biopsies, culture specimens. Surgery was performed under general anesthesia with no difficulties.

The sinonasal biopsies achieved in different sites and sized fragments. Specimens were fixed in formalin, then sent for histopathology and immunohistochemically analysis in our hospital.

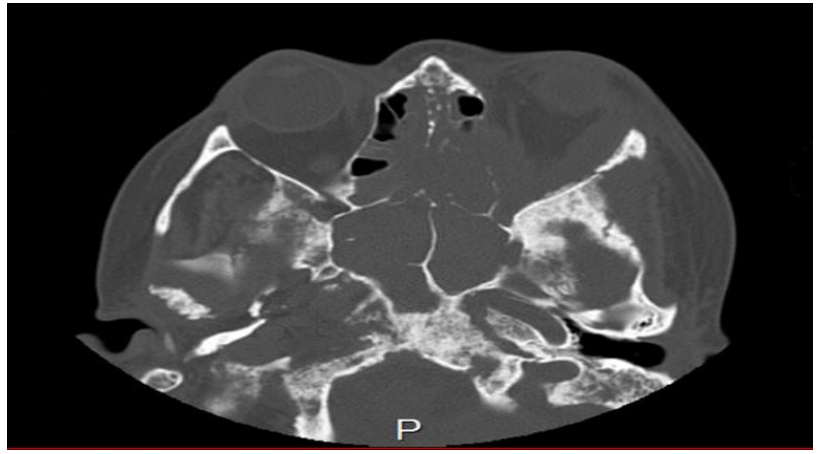

Figure 2: Bone window shows destruction of ethmoidal bone and complete opacification of sphenoid associated with left side sclerosis of skull base bone.

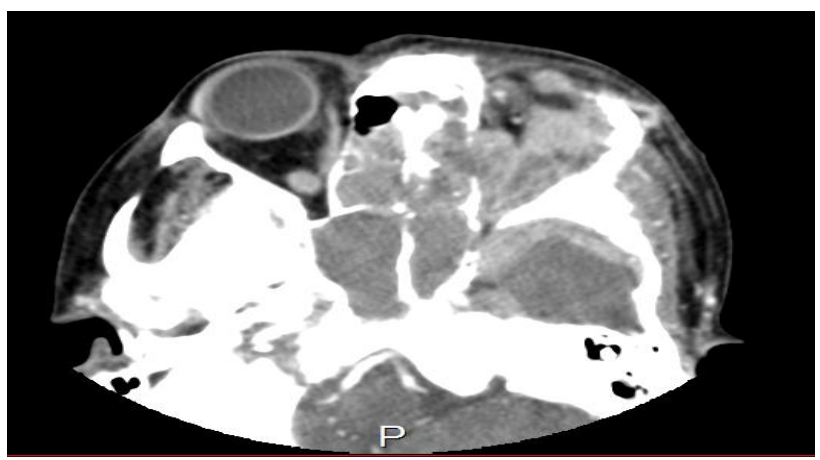

Figure 3: After i.v. contrast, it shows left intra orbital extension and left transcranial edema temporal lobe.

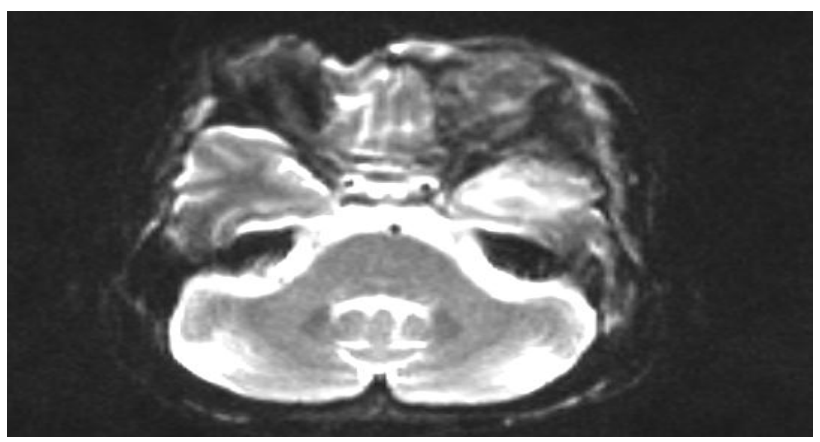

Figure 4: T2 MRI shows left intra orbital extension.

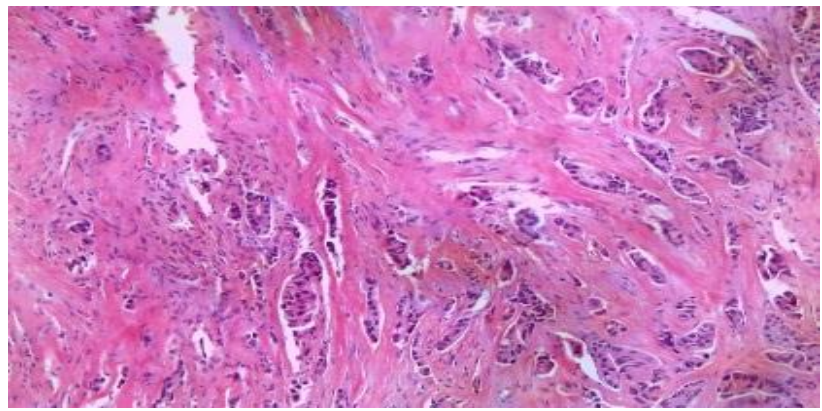

Figure 5: High power view clusters of malignant tumor cells in a background of desmoplastic stroma, H\&E. 


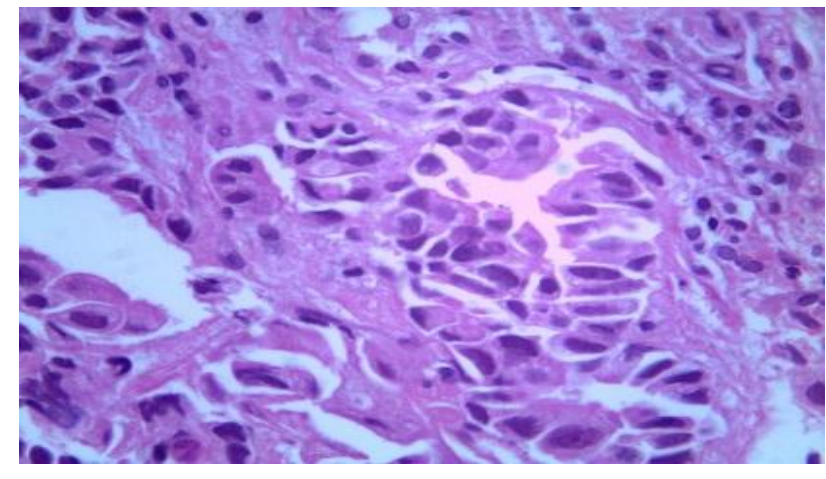

Figure 6: High power view cluster of tumor cells with moderate eosinophilic marked hyperchromatic pleomorphic nuclei, H\&E.

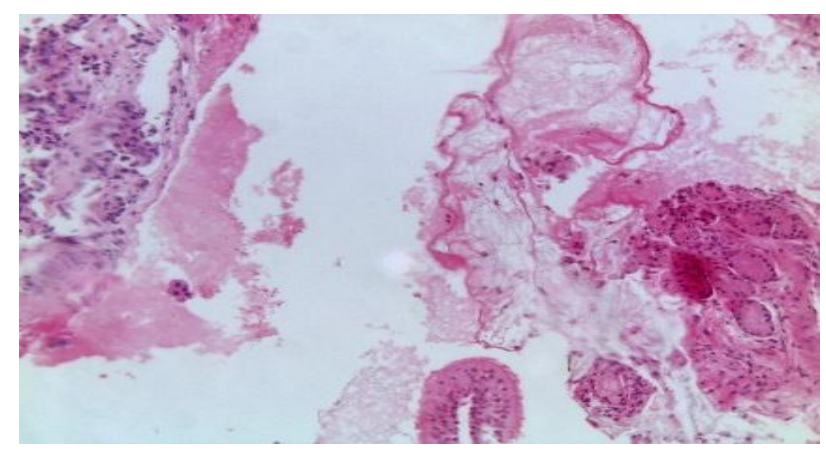

Figure 7: lower power view fragments of a normal tissue with normal mucus glands and normal ciliated respiratory epithelium beside this tissue there is a fibrous tissue infiltrated with clusters of malignant tumor cells, H\&E.

Multiple tissue fragments collected measured $1.5 \times 1 \times 0.5$ $\mathrm{cm}^{3}$, soft in consistency and gray/white in color as gross histological description. In addition, in the microscopic report there is a tumor cells arrange in small nests, cords and individually in background of desmoid fibrous stroma in addition few tubule like structures formed by tumor cells are noted Figure 5. The tumor cells are with hyperchromatic nuclei of marked atypia. In addition of pale eosinophilic cytoplasm Figure 6. Occasional abnormal mitosis is seen. Moreover, some fragments show a normal looking respiratory epithelium layer overlying the tumor with a focus of a normal looking mucous gland Figure 7. Small trabeculae of bone are also seen. Mild inflammatory infiltrate by lymphocyte and plasma cells are seen in one fragment, which contain a normal looking mucous glands in a background of mixed stroma and devoid of tumor cells. Estrogen receptors(ER), progesterone receptors (PR), gross cystic disease fluid protein-15 (GCDFP-15), cytokeratin-7 (CK7), cytokeratin-20 (CK20), transcription intermediary factor 1 (TIF-1), S-100 and p53 are used in immunohistochemistry panel. No reacting with the ER and PR with weak immunostaining for GCDFP-15. On the other hand, a positive immunostaining was found for cytokeratins with a high labelling index by $\mathrm{p}-53$ nuclear overexpression. On comparing to histopathological and immunohistochemistry report at 2010 for the primary breast carcinoma it showed the same characteristics as in the sinonasal histological and immunophenotypic study. Metastatic breast carcinoma was the final histopathological diagnosis. After final diagnosis, the patient was referred agained to the oncology Unit at high specialty hospital in Riyadh for palliative chemoradiotherapy.

\section{DISCUSSION}

Distant metastatic tumors to the head and neck uncommon. Moreover, metastatic tumors to paranasal sinuses are exclusively rare and it usually primary in origin. ${ }^{8,9}$ The common presenting symptoms of paranasal sinus metastasis mimic those of rhinosinusitis and therefore, can lead to a delay in diagnosis. The choice of appropriate treatment in such cases is extremely difficult since the surgical approach cannot be radical. Surgery usually being limited to obtaining a tumor biopsy for differential diagnosis. The vast majority of these patients undergo palliative radiotherapy in combination with chemotherapy. Bernstein et al. described the first cases reported in the literature in $1966 .^{5}$ The most known tumors to have a potential to give metastases in this area are from renal cancer. ${ }^{5}$ The most common sites for secondary breast cancer metastases are the lungs, liver, ovaries and adrenal glands. However, breast cancer can also give metastases to the paranasal sinuses. ${ }^{1,10-12}$ In the literature, only one case of metastatic melanoma to the ethmoid sinus has been reported. ${ }^{13}$ In-patient with renal cancer and frequent epistaxis should be suspected and roll out metastasis from renal cancer to sinonasal area. ${ }^{14}$ Loss of vision, decreased visual acuity, diplopia, and proptosis are the clinical symptoms of orbital affected by the metastatic lesion. ${ }^{15,16}$ the distant spread is unusual before locally spreads. ${ }^{17}$ Metastatic way to the paranasal sinuses until now it is unclear and there many controversial theories. Breast cancer spreads by both blood vessels and lymphatic channels. ${ }^{7,9}$ However, it can spread through vertebral venous plexus, prevertebral and epidural veins with innumerable intertwining vessels that communicate at every level, with the intercostal veins, venae cava, azygous vein and pelvic system., ${ }^{5,18}$ In addition, other theory suggested direct transcribrosal spread to ethmoidal sinus. ${ }^{1} \quad$ Final diagnosis is usually based on correlation between clinical, radiological, histopathological and immunohistochemistry findings. The main treatment of those patients with advanced breast carcinoma and metastatic to head and neck is palliative to improve or maintain their quality of life. ${ }^{17}$ Surgery usually being limited role only for tumour biopsy for differential diagnosis. By the way, the Local radiotherapy is the treatment of choice for pain relief. Preventing tumor growth and for organ function preservation with some conditions with chemotherapy. ${ }^{19,20}$ Systematic therapy with bevacizumab, trastuzumab and docetaxel are currently evaluated in therapeutic protocols. ${ }^{21}$ However, the breast cancer metastasis to paranasal sinuses considered very bad prognosis. By reviewed reported 
cases, most of those patients died in few months after diagnosis.

Funding: No funding sources

Conflict of interest: None declared

Ethical approval: Not required

\section{REFERENCES}

1. Monserez D, Vlaminck S, Kuhweide R, Casselman J. Symmetrical ethmoidal metastases from ductal carcinoma of the breast, suggesting transcribrosal spread. Acta Oto-rhinolaryngol. 2001;55:251-7.

2. Weber AL, Strnton AC. Malignant tumors of the paranasal sinuses: radiologic, clinical, and histopathologic evaluation of 200 cases. Head Neck Surg. 1984;6:761-76.

3. Sountoulides P, Metaxa L, Cindolo L. Atypical presentations and rare metastatic sites of renal cell carcinoma: a review of case reports. J Med Case Rep. 2011;5:429.

4. Evgeniou E, Menon KR, Jones GL, Whittet H, Williams W. Renal cell carcinoma metastasis to the paranasal sinuses and orbit. BMJ Case Rep. 2012;2012.

5. Bernstein JM, Montgomery MD, Balogh $\mathrm{K} \mathrm{Jr}$. Metastatic tumours to maxilla, nose, and paranasal sinuses. Laryngoscope. 1966;76:621-50.

6. Dineen MK, Pastore R, Emrich LJ, Huben RP. Results of surgical treatment of renal cell carcinoma with solitary metastasis. J Urol. 1988;140:2:277-9.

7. Batson OV. The function of the vertebral veins and their role in the spread of metastasis. Ann Surg. 1988;112:138-49.

8. Namad T, Benbrahim Z, Najib R, Mohammed A, Baggar S, Bouyahia N, Arifi S, Mellas N. Maxillofacial metastasis from breast cancer. Pan African Med J. 2014;19:156.

9. Marchioni D, Monzani D, Rossi G, Rivasi F, Presutti L. Breast carcinoma metastases in paranasal sinuses, a rare occurrence mimicking a primary nasal malignancy: Case report. Acta Otorhinolaryngol Ital. 2004;24(2):87-91.

10. Jortay AM. Metastatic tumors in oral cavity pharynx and paranasal sinuses. Acta Chir Belg. 1971;70(8):715-28.
11. Austin JR, Kershiznek MM, McGill D, Austin SG. Breast carcinoma metastatic to paranasal sinuses. Head Neck. 1995;17(2):161-5.

12. Myers EN. Metastatic carcinoma of the breast occurring in the frontal sinus. J Laryngol Otol. 1968;82(5):485-7

13. Bizon JG, Newman RK. Metastatic melanoma to ethmoid sinus. Arch Otol Head Neck Surg. 1986:112:664-7.

14. Yoshimura T, Suenaga T, Kozaki H, Oohashi N, Sakai N. A case of renal cell carcinoma revealed by metastasis to the nasal cavity and paranasal sinuses. Sapporo Hosp Med. 1989;49:31-5.

15. Barrs DM, McDonald TJ, Whisnant JP. Metastatic tumors to the sphenoid sinus. Laryngoscope. 1979;89:1239-43.

16. Bloch RS, Gartner S. The incidence of ocular metastatic carcinoma. Arch Ophthalmol. 1971;85:673-5.

17. Carnelio S, Rodrigues G. Maxillary Metastasis of Gastric Adenocarcinoma. Int J Oral-Med Sci. 2004;3(3):159-62.

18. Nahum AM, Bailey BJ. Malignant tumors metastatic to the paranasal sinuses. Laryngoscope. 1963;73:942-53.

19. Hirshberg A, Shnaiderman-Shapiro A, Kaplan I, Berger R. Metastatic tumours to the oral cavitypathogenesis and analysis of 673 cases. Oral Oncol. 2008;44(8):743-52.

20. AshabYamin MR, Kalantarhormozi A, Hamdamjo F, Razi Z. Breast ductal carcinoma metastasis to jaw bones: a case report. Novel Biomed. 2014;2(1):31-3.

21. Valachis A, Polyzos NP, Patsopoulos NA, Georgoulias V, Mavroudis D, Mauri D. Bevacizumab in metastatic breast cancer: a metaanalysis of randomized controlled trials. Breast Cancer Res Treat. 2010;122(1):1-7.

Cite this article as: Khurayzi T, Alshahrani A. Metastatic paranasal sinuses adenocarcinoma from breast carcinoma, a rare event: a case report. Int J Otorhinolaryngol Head Neck Surg 2017;3:1099-102. 